\title{
Symptomatic burden of COPD for patients receiving dual or triple therapy
}

This article was published in the following Dove Press journal: International journal of COPD

\author{
Stephanie Chen' \\ Mark Small ${ }^{2}$ \\ Leandro Lindner ${ }^{3}$ \\ Xiao $\mathrm{Xu}^{1,4}$ \\ 'Health Economics and Payer \\ Analytics, AstraZeneca, Gaithersburg, \\ MD, USA; ${ }^{2}$ Respiratory, Adelphi Real \\ World, Bollington, UK; ${ }^{3}$ Global Payer \\ Evidence and Pricing, AstraZeneca, \\ Cambridge, UK; ${ }^{4}$ Global Payer \\ Evidence and Pricing, AstraZeneca, \\ Gaithersburg, MD, USA
}

Background: COPD is associated with a large disease burden. The use of dual (two maintenance treatments) and triple (combination of any three treatments) therapy has shown efficacy for symptom relief; however, some patients with COPD remain symptomatic despite these therapies. This study assessed the scope and magnitude of the symptomatic burden for patients with COPD receiving dual or triple therapy.

Patients and methods: Cross-sectional data from three Adelphi COPD surveys (2013-2016) conducted in the USA, Europe, Japan, and China were analyzed for patients with COPD and forced expiratory volume in 1 second $\leq 65 \%$ receiving dual or triple therapy for $\geq 3$ months. Physicians completed clinical and disease characteristic forms for identified patients. Corresponding patients completed questionnaires that included validated survey instruments to assess adherence and symptom impact. Descriptive statistics are reported.

Results: Our analysis included 690 patients (mean age 68.2 years; $73.3 \%$ male); $41.4 \%$ and $58.6 \%$ were receiving dual and triple therapy, respectively. Most patients had dyspnea with substantial disability (modified Medical Research Council dyspnea scale rating $\geq 2,56.3 \%$; large health status impairment from symptoms, COPD Assessment Test score $>20,64.4 \%$ ). A large symptom burden was observed, even for patients highly adherent to treatment (Morisky Medication Adherence Scale 8, 30.3\% [185/612]), of whom 62.1\% still had a COPD Assessment Test score $>20$. Sensitivity analyses of patients regardless of their forced expiratory volume in 1 second status and of those receiving treatment for $>6$ months both reported similar results. Conclusion: Although patients who consult their physicians more frequently than average may be overrepresented because of the observational design of this study, we report that unmet needs remain for patients with COPD, despite the use of dual or triple therapy. A percentage of patients with COPD reported major symptom burden affecting their daily living and causing a large impairment in the health status, regardless of treatment adherence.

Keywords: COPD, burden of illness, triple therapy, dual therapy

\section{Introduction}

COPD is associated with a large disease burden. An estimated 328 million people worldwide have COPD, ${ }^{1}$ and the day-to-day impact of the disease can be debilitating for these patients. COPD is associated with significant health care costs of $\$ 18$ billion annually in the USA, and globally it accounts for 29.4 million years lost because of disability. ${ }^{1}$

The current Global Initiative for Chronic Obstructive Lung Disease (GOLD) guidelines grade the severity of COPD based on spirometry assessment to assess the severity of airflow limitation and use criteria based on symptoms and exacerbations in the previous year to estimate the risk of future exacerbations. ${ }^{2}$ GOLD recommends assessment of symptoms, including cough and/or sputum production, by using validated
Correspondence: Stephanie Chen AstraZeneca, I Medlmmune Way, Gaithersburg, MD 20878, USA

$\mathrm{Tel}+\mathrm{I} 3013982598$

Email stephanie.chen@astrazeneca.com 
patient questionnaires, such as the COPD Assessment Test (CAT) or the modified Medical Research Council (mMRC) dyspnea scale. ${ }^{2}$ This symptom assessment and history of exacerbations (which are indicative of symptom worsening) are important factors for predicting deterioration in lung function and likelihood of future exacerbations.

Either a short- or long-acting bronchodilator is the initial treatment option for relief of breathlessness for patients with COPD, with a long-acting bronchodilator recommended for patients with more persistent symptoms. ${ }^{2}$ Dual therapy (any combination of two maintenance treatments, including inhaled corticosteroids [ICS], long-acting $\beta_{2}$-agonists [LABA], or long-acting muscarinic antagonists [LAMA]) is recommended for patients with persistent breathlessness on monotherapy, and triple therapy (any combination of ICS, LABA, and LAMA) is recommended for patients who develop further exacerbations while on dual therapy. ${ }^{2}$ Both dual and triple therapy treatment regimens have been shown to improve the symptoms of patients with COPD. ${ }^{2,3}$ However, some patients receiving dual or triple therapy report still suffering from a considerable symptom burden that adversely affects their health-related quality of life (HRQOL) and leads to increased morbidity. ${ }^{4}$ For these patients, currently available medications may not be sufficient to provide relief from symptoms or to stabilize disease.

The aim of this study was to further assess the symptom burden of patients with COPD who were receiving dual or triple therapy. We sought to provide evidence of burden of disease in a real-world clinical setting to further characterize the current symptom burden and unmet treatment need for patients with COPD from Europe, Japan, China, and the USA.

\section{Patients and methods}

For these analyses, we used data from the Adelphi Real World Respiratory Disease Specific Programme (DSP). ${ }^{5}$ DSP is a real-world, point-in-time, patient record-based survey. The DSP provides impartial observations of realworld clinical practice from a physician and matched-patient viewpoint, and is an established method for investigating multicenter patient characteristics and treatment practices for common chronic diseases. DSPs in respiratory disease were conducted in Quarter 4 (Q4) 2013, Q4 2014/Quarter 1 (Q1) 2015, and Q4 2015/Q1 2016. The Respiratory DSPs collected information from patients diagnosed with COPD who were consulting their health care professionals for routine care.

The primary objective of this study was to assess the symptom burden of patients with COPD who were receiving dual or triple therapy and had a forced expiratory volume in 1 second $\left(\mathrm{FEV}_{1}\right)<65 \%$. Exploratory objectives included assessments for patients with low, moderate, or high treatment adherence and those with differing levels of lung function and length of dual or triple treatment.

All patients included in the Respiratory DSP provided written informed consent to participate. Because this study was a retrospective analysis of secondary data, we had no access to any medical or other patient records or to data sources containing patient or participating physician information. All patient management and treatment choices were at the discretion of the physician, and no tests or measurements were performed as part of the survey.

\section{Ethical approval}

The DSP was conducted as a survey that adhered to market research guidelines and the international code of conduct for observational research from the International Chamber of Commerce/European Society for Opinion and Marketing Research. ${ }^{6}$ Therefore, ethical approval was not necessary to obtain and was not sought. Patients provided informed consent to participate in the survey via a tick box on the front of the patient self-completion questionnaire. All data were anonymized and aggregated prior to receipt by Adelphi Real World.

\section{DSP survey population}

Primary care physicians and pulmonologists were qualified to participate in the Respiratory DSP if they were responsible for treatment of patients with COPD, qualified as a medical doctor 5-35 years ago, and saw $\geq 3$ patients with COPD per month.

\section{Study population}

A pooled data set from the three surveys was created to analyze cross-sectional data related to patient characteristics of a large, robust study population. Patients from Europe (France, Germany, Italy, Spain, and the UK), Japan, China, and the USA were included. The following inclusion criteria were used to select patients for the primary study population: physician-confirmed diagnosis of COPD; physicianrecorded post-bronchodilator $\mathrm{FEV}_{1} \leq 65 \%$ predicted (this requirement was removed in sensitivity analysis); and currently prescribed dual therapy (any combination of two maintenance treatments, including ICS, LABA, or LAMA) or triple therapy (any combination of ICS, LABA, and LAMA) for $\geq 3$ months. Patients declining to complete a patient self-completion questionnaire were excluded from 
the analysis to limit the amount of missing information for the study population.

\section{Study variables and analysis}

Participating physicians completed patient record forms for five consecutive consultations with patients with COPD. Physicians recorded demographics; clinical characteristics, such as exacerbation history; and treatment information. Patients completed disease-related questionnaires, including a patient self-completion questionnaire (detailing the nature of symptoms and the impact on daily activities), and the following validated questionnaires: Morisky Medication Adherence Scale 8 (MMAS-8), ${ }^{7-9} \mathrm{CAT},{ }^{10} \mathrm{mMRC}$ dyspnea scale, ${ }^{11}$ and Jenkins Sleep Evaluation Questionnaire (JSEQ). ${ }^{12}$ The MMAS- 8 consists of eight items that provide a score from 0 to 8; patient adherence to treatment is classified as low $(<6)$, moderate (6-7), or high (8). ${ }^{7}$ The CAT patient-reported outcome is an established tool for monitoring COPD. It consists of eight items, each with a 6-point differential scale that produces a score from 0 to 40 . A higher score indicates a more severe impact of COPD on the patient's life. ${ }^{10}$ The mMRC dyspnea scale consists of five statements about the impact of breathlessness and results in a grade from 0 to 4 , where grade 0 equates to the patient experiencing breathlessness only during strenuous exercise and grade 4 equates to the patient being too breathless to leave the house/breathless when dressing. ${ }^{11}$ An $\mathrm{mMRC} \geq 2$ is associated with a moderate to severe level of dyspnea. The JSEQ consists of four statements regarding sleep disturbance, which patients score by the frequency of experience; total scores range from 0 (no sleep disturbance) to 20 (22-28 days of disturbed sleep for all four statements). ${ }^{12}$

Each variable was described using the maximum available sample size; therefore, sample sizes varied from one variable to another, depending on the quantity of missing data. All analyses were conducted by the Adelphi Real World statistical department using Stata 14.2 or later. ${ }^{13}$ All code and result files were saved and are replicable. Descriptive statistics (univariate and bivariate) were used to examine symptom burden across a range of symptom-related characteristics.

\section{Sensitivity analysis}

In addition to the overall population, patient characteristics and symptom burden were also assessed for patients categorized by GOLD ABCD group (defined by exacerbation risk and CAT score $)^{2}$ to provide characterization based on symptoms and exacerbation risk, together with categorization based on treatment adherence (determined by the MMAS- 8 score). Patients with COPD on dual or triple therapy regardless of $\mathrm{FEV}_{1}$ status and those with $\mathrm{FEV}_{1} \leq 65 \%$ receiving dual or triple therapy for a minimum of 6 months were also assessed.

\section{Results Study population}

During the observation period (2013-2016), 7,094 patients were diagnosed with COPD and completed a patient selfcompletion questionnaire. Of these patients, 690 met the inclusion criteria for the primary study population. The main reason for exclusion was missing lung function data/ $\mathrm{FEV}_{1}>65 \%(\mathrm{n}=4,121)$; other patients were excluded because they had not been on dual or triple therapy for $>3$ months or they had missing duration data. Corresponding information relating to these patients was provided by 436 physicians. Removal of the inclusion criteria for $\mathrm{FEV}_{1}$ resulted in a population of 2,954 patients.

The mean age of the primary study population was 68.2 years; $73.3 \%$ were male, and $26.4 \%$ were current smokers (Table 1). Most patients had one or more comorbidity $(74.8 \%)$. Cardiovascular conditions were the most common concomitant condition; $64.6 \%$ of patients had concomitant hypertension (Table 1). Most patients were under a pulmonologist's care (77.1\%; Table 1$)$.

In the population of patients with COPD on dual or triple therapy for a minimum of 3 months regardless of $\mathrm{FEV}_{1}$ status, patient characteristics were similar to those of the primary study population (Table 1 ). The study population with $\mathrm{FEV}_{1} \leq 65 \%$ and on dual or triple therapy for a minimum of 6 months was also similar to the primary study population (Table 1).

\section{Treatment}

In the primary study population, $41.4 \%$ and $58.6 \%$ of patients were on dual and triple therapy, respectively (Table 1). ICS/ LABA (fixed-dosage combination) was the most common dual therapy (41.6\%) and ICS/LABA plus LAMA was the most common triple therapy $(88.9 \%)$ prescribed to patients. The percentages of patients receiving triple therapy in each of the GOLD ABCD categories were as follows: group A, 35.7\%; group B, 50.4\%; group C, 83.3\%; and group D, 66.6\%.

For the sensitivity analysis populations, $52.9 \%$ and $47.1 \%$ of the overall population regardless of the $\mathrm{FEV}_{1}$ status were receiving dual and triple therapy, respectively. Of patients with $\mathrm{FEV}_{1} \leq 65 \%$ on treatment for $\geq 6$ months, $41.3 \%$ and $58.7 \%$ were receiving dual and triple therapy, respectively (Table 1). 
Table I Patient demographics and baseline clinical characteristics

\begin{tabular}{|c|c|c|c|}
\hline & $\begin{array}{l}\text { Primary study } \\
\text { population } \\
(n=690)\end{array}$ & $\begin{array}{l}\text { Study population, } \\
\text { no FEV, criteria } \\
(n=2,954)\end{array}$ & $\begin{array}{l}\text { Study population, } \\
\text { dual/triple therapy } \\
\geq 6 \text { months }(n=606)\end{array}$ \\
\hline Mean age, years (SD) & $68.2(8.9)$ & $67.1(9.5)$ & $68.3(8.8)$ \\
\hline \multicolumn{4}{|l|}{ Sex, n (\%) } \\
\hline Male & $506(73.3)$ & $2,034(68.9)$ & $169(27.9)$ \\
\hline Race, n (\%) & $\mathrm{n}=688$ & $n=2,943$ & $\mathrm{n}=604$ \\
\hline White & $602(87.5)$ & $2,440(82.9)$ & $538(89.1)$ \\
\hline African American & $16(2.3)$ & $64(2.2)$ & $10(1.7)$ \\
\hline Hispanic/Latino & $18(2.6)$ & $72(2.4)$ & $14(2.3)$ \\
\hline Others & $52(7.6)$ & $367(12.4)$ & $42(7.0)$ \\
\hline Body mass index, $\mathrm{kg} / \mathrm{m}^{2}$ group, $\mathrm{n}(\%)$ & $\mathrm{n}=658$ & $n=2,838$ & $\mathrm{n}=574$ \\
\hline$<25$ & $249(37.8)$ & $1,093(38.5)$ & $212(36.9)$ \\
\hline $25-<27$ & $129(19.6)$ & $587(20.7)$ & $116(20.2)$ \\
\hline $27-<30$ & $145(22.0)$ & $619(21.8)$ & $|3|(22.8)$ \\
\hline $30+$ & $135(20.5)$ & $539(19.0)$ & $115(20.0)$ \\
\hline \multirow[t]{2}{*}{ Mean time since diagnosis, months (SD) } & $\mathrm{n}=623$ & $n=2,640$ & $\mathrm{n}=548$ \\
\hline & $77(66.7)$ & $68.5(64.2)$ & $77(66.9)$ \\
\hline Current therapy grouping, n (\%) & $\mathrm{n}=690$ & $n=2,954$ & $\mathrm{n}=606$ \\
\hline Dual & $286(4 I .4)$ & $\mathrm{I}, 564(52.9)$ & $250(4 I .3)$ \\
\hline Triple & $404(58.6)$ & $\mathrm{I}, 390(47.1)$ & $356(58.7)$ \\
\hline Dual therapy combinations, $\mathrm{n}(\%)$ & $\mathrm{n}=286$ & $n=1,564$ & $\mathrm{n}=250$ \\
\hline LABA+LAMA & $80(28.0)$ & $302(19.3)$ & $74(29.6)$ \\
\hline LABA/LAMA fixed-dosage combination & $16(5.6)$ & $54(3.5)$ & $48(19.2)$ \\
\hline ICS+LABA & $7(2.4)$ & $30(1.9)$ & $7(2.8)$ \\
\hline ICS/LABA fixed-dosage combination & $119(41.6)$ & $894(57.2)$ & $107(42.8)$ \\
\hline ICS+LAMA & $64(22.4)$ & $284(18.2)$ & $14(5.6)$ \\
\hline Triple therapy combinations ICS/LABA/LAMA \pm others, $n(\%)$ & $n=404$ & $\mathrm{n}=1,390$ & $n=356$ \\
\hline ICS+LABA/LAMA & $12(3.0)$ & $34(2.4)$ & $9(2.5)$ \\
\hline ICS/LABA+LAMA & $359(88.9)$ & $1,282(92.2)$ & $318(89.3)$ \\
\hline LABA+ICS+LAMA & $33(8.2)$ & $74(5.3)$ & $29(8.1)$ \\
\hline ICS dosage (grouped), n (\%) & $n=463$ & $n=2,026$ & $n=416$ \\
\hline Low & II $4(24.6)$ & $554(27.3)$ & $108(26.0)$ \\
\hline Medium & $191(41.3)$ & $862(42.5)$ & $168(40.4)$ \\
\hline High & $158(34.1)$ & $610(30.1)$ & $140(33.7)$ \\
\hline Physician responsible for treatment decisions, $\mathrm{n}(\%)$ & $\mathrm{n}=689$ & $\mathrm{n}=2,95 \mathrm{I}$ & $\mathrm{n}=605$ \\
\hline Pulmonologist only & $465(67.5)$ & $1,546(52.4)$ & $407(67.3)$ \\
\hline Primary care physician only & $149(21.6)$ & $1,079(36.6)$ & $133(22.0)$ \\
\hline Primary care physician+pulmonologist & $66(9.6)$ & $298(10.1)$ & $56(9.3)$ \\
\hline Other & $9(1.3)$ & $28(0.9)$ & $9(1.5)$ \\
\hline MMAS-8, n (\%) & $\mathrm{n}=613$ & $n=2,616$ & $n=539$ \\
\hline Low, $<6$ & $209(34.1)$ & $936(35.8)$ & $180(33.4)$ \\
\hline Moderate, 6-7 & $219(35.7)$ & $845(32.3)$ & $193(35.8)$ \\
\hline High, 8 & $185(30.2)$ & $835(31.9)$ & $166(30.8)$ \\
\hline \multirow[t]{2}{*}{ Mean post-bronchodilator $\mathrm{FEV}_{1}$, \% predicted (SD) } & $\mathrm{n}=690$ & $\mathrm{n}=1,379$ & $\mathrm{n}=606$ \\
\hline & $50.27(11.6)$ & $63.49(16.4)$ & $49.94(11.7)$ \\
\hline \multirow[t]{2}{*}{ Exacerbations experienced in last 12 months, mean (SD) } & $\mathrm{n}=670$ & $n=2,880$ & $\mathrm{n}=587$ \\
\hline & $1.6(1.6)$ & $1.3(1.5)$ & $1.6(1.6)$ \\
\hline Smoking status, n (\%) & $\mathrm{n}=666$ & $n=2,869$ & $\mathrm{n}=584$ \\
\hline Ex-smoker & $465(69.8)$ & $1,824(63.6)$ & $154(26.4)$ \\
\hline Current smoker & $176(26.4)$ & $780(27.2)$ & $406(69.5)$ \\
\hline Has never smoked & $25(3.8)$ & $265(9.2)$ & $24(4.1)$ \\
\hline Cardiovascular conditions, n (\%) & $\mathrm{n}=642$ & $n=2,7 \mid 4$ & $n=564$ \\
\hline Any & $503(78.3)$ & $1,987(73.2)$ & $444(78.7)$ \\
\hline Hypertension & $415(64.6)$ & $1,636(60.3)$ & $366(64.9)$ \\
\hline Elevated cholesterol/hyperlipidemia & $214(33.3)$ & $833(30.7)$ & $184(32.6)$ \\
\hline
\end{tabular}


Table I (Continued)

\begin{tabular}{|c|c|c|c|}
\hline & $\begin{array}{l}\text { Primary study } \\
\text { population } \\
(\mathrm{n}=690)\end{array}$ & $\begin{array}{l}\text { Study population, } \\
\text { no FEV, criteria } \\
(n=2,954)\end{array}$ & $\begin{array}{l}\text { Study population, } \\
\text { dual/triple therapy } \\
\geq 6 \text { months }(n=606)\end{array}$ \\
\hline Concomitant conditions $\geq 5 \%$ of patients, $n(\%)$ & $n=620$ & $n=2,666$ & $n=546$ \\
\hline Diabetes & $129(20.8)$ & $463(17.4)$ & II 4 (20.9) \\
\hline Depression & $92(14.8)$ & $30 \mathrm{I}(1 \mathrm{I} .3)$ & $81(14.8)$ \\
\hline Gastroesophageal reflux disease & $84(13.5)$ & $330(12.4)$ & $76(13.9)$ \\
\hline Prostate disorder & $79(12.7)$ & $289(10.8)$ & $67(12.3)$ \\
\hline Anxiety & $72(\mathrm{I} I .6)$ & $323(12.1)$ & $66(12.1)$ \\
\hline Obesity & $72(11.6)$ & $253(9.5)$ & $64(11.7)$ \\
\hline Arthritis & $61(9.8)$ & $312(11.7)$ & $60(11.0)$ \\
\hline Osteoporosis & $53(8.5)$ & $198(7.4)$ & $48(8.8)$ \\
\hline Sleep apnea & $39(6.3)$ & $105(3.9)$ & $33(6.0)$ \\
\hline Dyspepsia/stomach pain & $37(6.0)$ & $162(6.1)$ & $34(6.2)$ \\
\hline
\end{tabular}

Notes: aLow, 100-250 $\mu \mathrm{g}$ fluticasone; medium, $>250-500 \mu \mathrm{g}$ fluticasone; high, $>500 \mu \mathrm{g}$ fluticasone. Use of the ${ }^{\circ M M A S}$ is protected by US copyright laws. Permission for use is required. A license agreement is available from Donald E Morisky, ScD, ScM, MSPH, Professor, Department of Community Health Sciences, UCLA School of Public Health, 650 Charles E. Young Drive South, Los Angeles, CA 90095- 1772.

Abbreviations: FEV ${ }_{1}$, forced expiratory volume in I second; ICS, inhaled corticosteroids; LABA, long-acting $\beta_{2}$-agonists; LAMA, long-acting muscarinic antagonists; MMAS-8, Morisky Medication Adherence Scale 8.

\section{Treatment adherence}

In the primary study population, 613 patients had completed MMAS-8; 185 patients were highly adherent to treatment $(30.2 \%)$. The mean age of patients highly adherent to treatment was 67.4 years; $72.4 \%$ were male, $26.8 \%$ were current smokers, and $68 \%$ suffered from concomitant hypertension. These patient characteristics were similar to those of the overall patient population.

\section{Timing of symptoms and frequency of reliever usage}

In the primary study population, symptoms occurred most frequently during winter $(91.7 \%)$ and autumn $(62.0 \%)$ months (Table 2). Of these patients, $56.7 \%$ reported that their symptoms occurred mainly during daytime hours, whereas $38.5 \%$ of other patients had equal daytime and nighttime occurrences. Use of a reliever inhaler at least once a week was reported by $67.1 \%$ of patients. Patients with greater maintenance medication adherence tended to use their reliever inhaler less frequently than those with lower adherence (Table 3 ). However, of patients with high adherence, 59.4\% still reported using their reliever at least once a week and $33.3 \%$ used a reliever inhaler three to six times a week or every day.

\section{Symptom burden}

In the primary study population, $45.1 \%$ were dissatisfied with their treatment but considered that they were receiving the best possible control that they could, and $11.1 \%$ were dissatisfied but believed that better control was possible (Table 2).
Shortness of breath during exertion/exercise was the most common troublesome symptom reported by patients (52.3\%; primary study population) during the preceding 4 weeks. Many patients reported experiencing a constant lack of energy (44.9\%), tiredness through lack of sleep (30.6\%), and were scared or worried about their own condition (31.1\%; Table 2). More than half of the patients also reported that COPD at least occasionally affected their leisure/personal time (69.8\%) and work (55.9\% [if in work]; Table 2). Physician-reported perceived impact of COPD on the patient's HRQOL results was similar to that self-reported by patients (Table 4 ), as was the one reported by patients in the study population, regardless of the $\mathrm{FEV}_{1}$ status or duration of treatment (Table 2). In the high-treatment adherence category, $58.9 \%$ of patients also reported that they experienced shortness of breath during exertion/exercise as their most common symptom experienced in the previous 4 weeks and that they experienced a constant lack of energy (42.2\%).

More than half of the patients in the primary study population had CAT scores $>20$ (64.4\%; Table 2; Figure 1) from a possible total score of 40 , indicating a large or very large impact of COPD on the patient's health status. ${ }^{14}$ More than $50 \%$ of patients had mMRC dyspnea scale scores $\geq 2$, indicating that many patients suffer a degree of breathlessness. ${ }^{15}$ The mean JSEQ score was 6.1 out of a possible total score of 20, indicative of experiencing sleep disturbance (Table 2). ${ }^{12}$ Similar results were observed for the study population, regardless of the $\mathrm{FEV}_{1}$ level or duration of treatment (Table 2). For patients categorized by GOLD ABCD group, $>50 \%$ of patients in GOLD groups B and D had CAT 
Table 2 Patient-reported symptom burden and health-related quality of life (patient self-completion questionnaire assessed)

\begin{tabular}{|c|c|c|c|}
\hline & $\begin{array}{l}\text { Primary } \\
\text { study } \\
\text { population }\end{array}$ & $\begin{array}{l}\text { Study } \\
\text { population, no } \\
\text { FEV, criteria }\end{array}$ & $\begin{array}{l}\text { Study population, } \\
\text { dual/triple therapy } \\
\text { for } \geq 6 \text { months }\end{array}$ \\
\hline Time of day of symptoms, $\mathrm{n}(\%)$ & $n=671$ & $n=2,87 \mid$ & $n=589$ \\
\hline Daytime only & $103(15.4)$ & $45 \mathrm{I}(15.7)$ & $84(14.3)$ \\
\hline Primarily daytime & $277(4 I .3)$ & $1,283(44.7)$ & $250(42.4)$ \\
\hline Equally day and night & $258(38.5)$ & $939(32.7)$ & $227(38.5)$ \\
\hline Primarily nighttime & $29(4.3)$ & $183(6.4)$ & $25(4.2)$ \\
\hline Nighttime only & $4(0.6)$ & $15(0.5)$ & $3(0.5)$ \\
\hline Time of year of symptoms, $n$ (\%) & $n=424$ & $\mathrm{n}=1,742$ & $\mathrm{n}=375$ \\
\hline Spring & 143 (33.7) & $553(31.7)$ & $130(34.7)$ \\
\hline Summer & $124(29.2)$ & $424(24.3)$ & $115(30.7)$ \\
\hline Autumn & $263(62.0)$ & $1,015(58.3)$ & $235(62.7)$ \\
\hline Winter & $389(91.7)$ & I,525 (87.5) & $34 \mid(90.9)$ \\
\hline Most troublesome symptom in the past 4 weeks, $\mathrm{n}(\%)$ & $\mathrm{n}=623$ & $\mathrm{n}=2,557$ & $n=551$ \\
\hline No symptoms & $\mathrm{I}(0.2)$ & $14(0.5)$ & $\mathrm{I}(0.2)$ \\
\hline Shortness of breath when resting & $78(12.5)$ & $233(9.1)$ & $67(12.2)$ \\
\hline Shortness of breath during exertion/exercise & $326(52.3)$ & $\mathrm{I}, 165(45.6)$ & $291(52.8)$ \\
\hline Shortness of breath when exposed to trigger & $14(2.2)$ & $61(2.4)$ & $13(2.4)$ \\
\hline Wheezing & $28(4.5)$ & $138(5.4)$ & $25(4.5)$ \\
\hline Productive cough/sputum & $95(15.2)$ & $510(19.9)$ & $81(14.7)$ \\
\hline Dry cough & $20(3.2)$ & I7I (6.7) & $18(3.3)$ \\
\hline Coughing up blood & $2(0.3)$ & $20(0.8)$ & $2(0.4)$ \\
\hline Regular clearing of throat & $7(1.1)$ & $45(1.8)$ & $7(1.3)$ \\
\hline A tight feeling in the chest & $34(5.5)$ & $119(4.7)$ & $3 \mid(5.6)$ \\
\hline Bronchospasm/sudden chest tightening & $18(2.9)$ & $81(3.2)$ & $15(2.7)$ \\
\hline Impact of COPD on getting up and ready for the day, $\mathrm{n}(\%)$ & $n=674$ & $\mathrm{n}=2,877$ & $n=591$ \\
\hline No impact & $95(14.1)$ & $640(22.2)$ & $81(13.7)$ \\
\hline Rarely impacts & $164(24.3)$ & $826(28.7)$ & $143(24.2)$ \\
\hline Occasionally impacts & $243(36.1)$ & $901(31.3)$ & $218(36.9)$ \\
\hline Frequently impacts & $137(20.3)$ & $402(14.0)$ & $118(20.0)$ \\
\hline Constantly impacts & $35(5.2)$ & $108(3.8)$ & $31(5.2)$ \\
\hline Impact of COPD on personal relationships, $\mathrm{n}(\%)$ & $\mathrm{n}=668$ & $\mathrm{n}=2,855$ & $n=585$ \\
\hline No impact & $101(15.1)$ & $613(2 \mid .5)$ & $90(15.4)$ \\
\hline Rarely impacts & $194(29.0)$ & $983(34.4)$ & $169(28.9)$ \\
\hline Occasionally impacts & $242(36.2)$ & $892(31.2)$ & $212(36.2)$ \\
\hline Frequently impacts & $103(15.4)$ & $300(10.5)$ & $89(15.2)$ \\
\hline Constantly impacts & $28(4.2)$ & $67(2.3)$ & $25(4.3)$ \\
\hline Impact of COPD on leisure/personal time, $\mathrm{n}(\%)$ & $\mathrm{n}=663$ & $\mathrm{n}=2,843$ & $n=581$ \\
\hline No impact & $53(8.0)$ & $370(13.0)$ & $47(8.1)$ \\
\hline Rarely impacts & $147(22.2)$ & $778(27.4)$ & $127(21.9)$ \\
\hline Occasionally impacts & $248(37.4)$ & $1,035(36.4)$ & $219(37.7)$ \\
\hline Frequently impacts & $168(25.3)$ & $546(19.2)$ & $146(25.1)$ \\
\hline Constantly impacts & $47(7.1)$ & $114(4.0)$ & $42(7.2)$ \\
\hline Impact of COPD on work (if in work), $n$ (\%) & $n=145$ & $n=740$ & $n=120$ \\
\hline No impact & $28(19.3)$ & $|7|(23.1)$ & $22(18.3)$ \\
\hline Rarely impacts & $36(24.8)$ & $264(35.7)$ & $33(27.5)$ \\
\hline Occasionally impacts & $53(36.6)$ & $218(29.5)$ & $48(40.0)$ \\
\hline Frequently impacts & $24(16.6)$ & $73(9.9)$ & $16(13.3)$ \\
\hline Constantly impacts & $4(2.8)$ & 14 (I.9) & $\mathrm{I}(0.8)$ \\
\hline Impact of COPD on sleep, $\mathrm{n}(\%)$ & $\mathrm{n}=668$ & $\mathrm{n}=2,845$ & $\mathrm{n}=585$ \\
\hline No impact & $116(17.4)$ & $577(20.3)$ & $103(17.6)$ \\
\hline Rarely impacts & $198(29.6)$ & $976(34.3)$ & I 74 (29.7) \\
\hline Occasionally impacts & $221(33.1)$ & $877(30.8)$ & $195(33.3)$ \\
\hline Frequently impacts & $110(16.5)$ & $356(12.5)$ & $93(15.9)$ \\
\hline Constantly impacts & $23(3.4)$ & $59(2.1)$ & $20(3.4)$ \\
\hline
\end{tabular}


Table 2 (Continued)

\begin{tabular}{|c|c|c|c|}
\hline & $\begin{array}{l}\text { Primary } \\
\text { study } \\
\text { population }\end{array}$ & $\begin{array}{l}\text { Study } \\
\text { population, no } \\
\text { FEV, criteria } \\
\end{array}$ & $\begin{array}{l}\text { Study population, } \\
\text { dual/triple therapy } \\
\text { for } \geq 6 \text { months }\end{array}$ \\
\hline Satisfaction with current control of COPD, n (\%) & $\mathrm{n}=506$ & $\mathrm{n}=2,198$ & $n=44 \mid$ \\
\hline Satisfied & $222(43.9)$ & $\mathrm{I}, 225(55.7)$ & $201(45.6)$ \\
\hline Dissatisfied, best possible control & $228(45.1)$ & $773(35.2)$ & $196(44.4)$ \\
\hline Dissatisfied, better control possible & $56(I I . I)$ & $200(9.1)$ & $44(10.0)$ \\
\hline Feelings experienced, $\mathrm{n}(\%)$ & $\mathrm{n}=666$ & $\mathrm{n}=2,878$ & $\mathrm{n}=585$ \\
\hline Constant lack of energy & $299(44.9)$ & $\mathrm{I}, 043(36.2)$ & $266(45.5)$ \\
\hline Tiredness through lack of sleep & $204(30.6)$ & $857(29.8)$ & $178(30.4)$ \\
\hline Sickness & $48(7.2)$ & $258(9.0)$ & $33(5.6)$ \\
\hline Nervousness or anxiety & $179(26.9)$ & $681(23.7)$ & $155(26.5)$ \\
\hline Feelings of sadness or depression & $134(20.1)$ & $409(14.2)$ & $117(20.0)$ \\
\hline Difficulty expressing your feelings & $63(9.5)$ & $215(7.5)$ & $54(9.2)$ \\
\hline Embarrassment about your condition & $125(18.8)$ & $390(13.6)$ & $110(18.8)$ \\
\hline Scared and worried about your condition & $207(31.1)$ & $719(25.0)$ & $180(30.8)$ \\
\hline Feelings of irritability & $164(24.6)$ & $625(21.7)$ & $145(24.8)$ \\
\hline None of them & $124(18.6)$ & $709(24.6)$ & $109(18.6)$ \\
\hline Reliever inhaler usage, $\mathrm{n}(\%)$ & $\mathrm{n}=654$ & $\mathrm{n}=2,785$ & $\mathrm{n}=572$ \\
\hline I am not prescribed a reliever inhaler & $42(6.4)$ & $392(14.1)$ & $38(6.6)$ \\
\hline Not at all & $74(11.3)$ & $442(15.9)$ & 67 (11.7) \\
\hline Less than once a week & $99(15.1)$ & $467(16.8)$ & $84(14.7)$ \\
\hline Once or twice a week & 191 (29.2) & $657(23.6)$ & $175(30.6)$ \\
\hline 3-6 times a week & III (I7.0) & $379(13.6)$ & $95(16.6)$ \\
\hline Every day & 137 (20.9) & $448(16.1)$ & $113(19.8)$ \\
\hline Modified Medical Research Council dyspnea scale, n (\%) & $\mathrm{n}=650$ & $\mathrm{n}=2,755$ & $\mathrm{n}=568$ \\
\hline I only get breathless with strenuous exercise (grade 0 ) & $64(9.8)$ & $492(17.9)$ & $56(9.9)$ \\
\hline $\begin{array}{l}\text { I get short of breath when hurrying on level ground or } \\
\text { walking up a slight hill (grade I) }\end{array}$ & $220(33.8)$ & $1,076(39.1)$ & $196(34.5)$ \\
\hline $\begin{array}{l}\text { On level ground, I walk slower than people of the same } \\
\text { age because of my breathlessness, or have to stop for } \\
\text { breath when walking at my own pace (grade 2) }\end{array}$ & $206(31.7)$ & $734(26.6)$ & $179(31.5)$ \\
\hline $\begin{array}{l}\text { I stop for breath after walking for a few minutes on level } \\
\text { ground (grade 3) }\end{array}$ & $114(17.5)$ & $338(12.3)$ & $98(17.3)$ \\
\hline $\begin{array}{l}\text { I am too breathless to leave the house or I am } \\
\text { breathless when dressing (grade 4) }\end{array}$ & $46(7.1)$ & $115(4.2)$ & $39(6.9)$ \\
\hline \multirow[t]{2}{*}{ COPD Assessment Test, mean (SD) } & $\mathrm{n}=648$ & $n=2,768$ & $\mathrm{n}=569$ \\
\hline & $23.1(7.9)$ & $21.2(8.0)$ & $22.9(7.7)$ \\
\hline COPD Assessment Test (grouped), n (\%) & $\mathrm{n}=648$ & $n=2,768$ & $\mathrm{n}=569$ \\
\hline $0-9$ & $43(6.6)$ & $254(9.2)$ & $36(6.3)$ \\
\hline $10-20$ & $188(29.0)$ & $971(35.1)$ & $175(30.8)$ \\
\hline $21-30$ & $298(46.0)$ & $1,213(43.8)$ & $261(45.9)$ \\
\hline$>30$ & $119(18.4)$ & $330(11.9)$ & $97(I 7.0)$ \\
\hline \multirow[t]{2}{*}{ Jenkins Sleep Evaluation Questionnaire, mean (SD) } & $\mathrm{n}=628$ & $\mathrm{n}=2,580$ & $n=559$ \\
\hline & $6.1(5.0)$ & $5.4(4.6)$ & $6.0(5.0)$ \\
\hline
\end{tabular}

Abbreviation: $\mathrm{FEV}_{1}$, forced expiratory volume in I second.

scores $>20$ (57.9\% and $78.5 \%$, respectively) and $>50 \%$ of patients in GOLD group D had mMRC dyspnea scale scores $\geq 2$ (Table 5 ).

Although there was some improvement in patient burden with increased medication adherence in the primary study population, substantial burden remained for patients who were most adherent. More than half of these patients (64.2\%) had a CAT score $>20$ (Table 3; Figure 1) and 54.2\% had an mMRC dyspnea scale score of $\geq 2$ (Table 3 ).

\section{Discussion}

The results of this analysis, using data collected via physician- and patient-reported questionnaires, highlight the large symptom burden experienced by patients with COPD. 
Table 3 Symptom burden and health-related quality of life by MMAS-8 (primary study population)

\begin{tabular}{|c|c|c|c|c|c|c|}
\hline \multirow[t]{2}{*}{ MMAS-8 } & \multicolumn{3}{|c|}{ Physician reported } & \multicolumn{3}{|c|}{ Patient reported } \\
\hline & $\begin{array}{l}\text { Low } \\
(n=209)\end{array}$ & $\begin{array}{l}\text { Moderate } \\
(n=2 \mid 9)\end{array}$ & $\begin{array}{l}\text { High } \\
(n=\mid 85)\end{array}$ & $\begin{array}{l}\text { Low } \\
(n=209)\end{array}$ & $\begin{array}{l}\text { Moderate } \\
(n=2 \mid 9)\end{array}$ & $\begin{array}{l}\text { High } \\
(n=\mid 85)\end{array}$ \\
\hline Impact of COPD on getting up and ready for the day, $\mathrm{n}(\%)$ & $\mathrm{n}=209$ & $\mathrm{n}=219$ & $\mathrm{n}=185$ & $\mathrm{n}=205$ & $\mathrm{n}=217$ & $n=183$ \\
\hline No impact & $17(8.1)$ & $33(15.1)$ & $22(11.9)$ & $22(10.7)$ & $29(13.4)$ & $30(16.4)$ \\
\hline Rarely impacts & $64(30.6)$ & $56(25.6)$ & $43(23.2)$ & $59(28.8)$ & $5 I(23.5)$ & $40(21.9)$ \\
\hline Occasionally impacts & $68(32.5)$ & $66(30.1)$ & $68(36.8)$ & $74(36.1)$ & $79(36.4)$ & $63(34.4)$ \\
\hline Frequently impacts & $46(22.0)$ & $55(25.1)$ & $42(22.7)$ & $39(19.0)$ & $46(21.2)$ & $40(21.9)$ \\
\hline Constantly impacts & $14(6.7)$ & $9(4.1)$ & $10(5.4)$ & II (5.4) & $12(5.5)$ & $10(5.5)$ \\
\hline Impact of COPD on personal relationships, $n$ (\%) & $\mathrm{n}=208$ & $n=216$ & $n=184$ & $\mathrm{n}=207$ & $\mathrm{n}=214$ & $n=179$ \\
\hline No impact & $16(7.7)$ & $23(10.6)$ & $37(20.1)$ & $25(12.1)$ & $30(14.0)$ & $29(16.2)$ \\
\hline Rarely impacts & $7 I(34.1)$ & $80(37.0)$ & $49(26.6)$ & $54(26.1)$ & $66(30.8)$ & $58(32.4)$ \\
\hline Occasionally impacts & $79(38.0)$ & $65(30.1)$ & $72(39.1)$ & $78(37.7)$ & 7I (33.2) & $70(39.1)$ \\
\hline Frequently impacts & $34(16.3)$ & $44(20.4)$ & $21(11.4)$ & $38(18.4)$ & $37(17.3)$ & $18(10.1)$ \\
\hline Constantly impacts & $8(3.8)$ & $4(1.9)$ & $5(2.7)$ & $12(5.8)$ & $10(4.7)$ & $4(2.2)$ \\
\hline Impact of COPD on leisure/personal time, $n(\%)$ & $\mathrm{n}=208$ & $\mathrm{n}=218$ & $\mathrm{n}=185$ & $\mathrm{n}=204$ & $n=213$ & $n=180$ \\
\hline No impact & $6(2.9)$ & $13(6.0)$ & $14(7.6)$ & $14(6.9)$ & $10(4.7)$ & $16(8.9)$ \\
\hline Rarely impacts & $45(21.6)$ & $48(22.0)$ & $47(25.4)$ & $44(21.6)$ & $53(24.9)$ & $4 I(22.8)$ \\
\hline Occasionally impacts & $84(40.4)$ & $72(33.0)$ & $63(34.1)$ & $76(37.3)$ & $81(38.0)$ & $67(37.2)$ \\
\hline Frequently impacts & $58(27.9)$ & $70(32.1)$ & $49(26.5)$ & $56(27.5)$ & $50(23.5)$ & $44(24.4)$ \\
\hline Constantly impacts & $15(7.2)$ & $15(6.9)$ & $12(6.5)$ & $14(6.9)$ & $19(8.9)$ & $12(6.7)$ \\
\hline Impact of COPD on work (if in work), $n$ (\%) & $\mathrm{n}=44$ & $n=4 \mid$ & $n=37$ & $\mathrm{n}=47$ & $n=4 I$ & $n=41$ \\
\hline No impact & $6(13.6)$ & $5(12.2)$ & $5(13.5)$ & $9(19.1)$ & $8(19.5)$ & $9(22.0)$ \\
\hline Rarely impacts & $10(22.7)$ & $14(34.1)$ & II (29.7) & $7(14.9)$ & $13(31.7)$ & $12(29.3)$ \\
\hline Occasionally impacts & $12(27.3)$ & $13(31.7)$ & $16(43.2)$ & $21(44.7)$ & II (26.8) & $15(36.6)$ \\
\hline Frequently impacts & $14(31.8)$ & $6(14.6)$ & $3(8.1)$ & $8(17.0)$ & $7(17.1)$ & $5(12.2)$ \\
\hline Constantly impacts & $2(4.5)$ & $3(7.3)$ & $2(5.4)$ & $2(4.3)$ & $2(4.9)$ & $0(0.0)$ \\
\hline Impact of COPD on sleep, $\mathrm{n}(\%)$ & $\mathrm{n}=208$ & $\mathrm{n}=218$ & $\mathrm{n}=184$ & $\mathrm{n}=207$ & $\mathrm{n}=215$ & $n=180$ \\
\hline No impact & $21(10.1)$ & $39(17.9)$ & $40(21.7)$ & $29(14.0)$ & $38(17.7)$ & $37(20.6)$ \\
\hline Rarely impacts & $70(33.7)$ & $75(34.4)$ & $59(32.1)$ & $63(30.4)$ & $62(28.8)$ & $54(30.0)$ \\
\hline Occasionally impacts & $67(32.2)$ & $67(30.7)$ & $59(32.1)$ & $68(32.9)$ & $76(35.3)$ & $59(32.8)$ \\
\hline Frequently impacts & $43(20.7)$ & $32(14.7)$ & $23(12.5)$ & $38(18.4)$ & $31(14.4)$ & $26(14.4)$ \\
\hline Constantly impacts & $7(3.4)$ & $5(2.3)$ & $3(1.6)$ & $9(4.3)$ & $8(3.7)$ & $4(2.2)$ \\
\hline Satisfaction with current control of COPD, n (\%) & & & & $\mathrm{n}=145$ & $\mathrm{n}=158$ & $n=148$ \\
\hline Satisfied & & & & $56(38.6)$ & $66(41.8)$ & $78(52.7)$ \\
\hline Dissatisfied, best possible control & & & & $66(45.5)$ & $81(5 \mid .3)$ & $58(39.2)$ \\
\hline Dissatisfied, better control possible & & & & $23(15.9)$ & II (7.0) & $12(8.1)$ \\
\hline Feelings experienced, $n(\%)$ & & & & $n=206$ & $n=216$ & $n=180$ \\
\hline Constant lack of energy & & & & $90(43.7)$ & $102(47.2)$ & $76(42.2)$ \\
\hline Tiredness through lack of sleep & & & & $72(35.0)$ & $65(30.1)$ & $45(25.0)$ \\
\hline Sickness & & & & $20(9.7)$ & $15(6.9)$ & $6(3.3)$ \\
\hline Nervousness or anxiety & & & & $64(31.1)$ & $62(28.7)$ & $41(22.8)$ \\
\hline Feelings of sadness or depression & & & & $49(23.8)$ & $47(21.8)$ & $31(17.2)$ \\
\hline Difficulty expressing your feelings & & & & $28(13.6)$ & $17(7.9)$ & $12(6.7)$ \\
\hline Embarrassment about your condition & & & & $4 I(19.9)$ & $51(23.6)$ & $29(16.1)$ \\
\hline Scared and worried about your condition & & & & $67(32.5)$ & $73(33.8)$ & $49(27.2)$ \\
\hline Feelings of irritability & & & & $64(31.1)$ & $50(23.1)$ & $37(20.6)$ \\
\hline None of them & & & & $27(13.1)$ & $41(19.0)$ & $50(27.8)$ \\
\hline Reliever inhaler usage, n (\%) & & & & $n=203$ & $n=208$ & $n=180$ \\
\hline I am not prescribed a reliever inhaler & & & & $9(4.4)$ & $15(7.2)$ & $15(8.3)$ \\
\hline Not at all & & & & $10(4.9)$ & $30(14.4)$ & $29(16.1)$ \\
\hline Less than once a week & & & & $26(12.8)$ & $32(15.4)$ & $29(16.1)$ \\
\hline Once or twice a week & & & & $76(37.4)$ & $52(25.0)$ & $47(26.1)$ \\
\hline $3-6$ times a week & & & & $39(19.2)$ & $28(13.5)$ & $29(16.1)$ \\
\hline Every day & & & & $43(21.2)$ & $5 \mathrm{I}(24.5)$ & $31(17.2)$ \\
\hline Modified Medical Research Council dyspnea scale, n (\%) & & & & $n=199$ & $n=210$ & $\mathrm{n}=175$ \\
\hline I only get breathless with strenuous exercise (grade 0 ) & & & & $17(8.5)$ & $21(10.0)$ & $20(11.4)$ \\
\hline $\begin{array}{l}\text { I get short of breath when hurrying on level ground or } \\
\text { walking up a slight hill (grade I) }\end{array}$ & & & & $66(33.2)$ & $72(34.3)$ & $60(34.3)$ \\
\hline
\end{tabular}


Table 3 (Continued)

\begin{tabular}{|c|c|c|c|c|c|c|}
\hline \multirow[t]{2}{*}{ MMAS-8 } & \multicolumn{3}{|c|}{ Physician reported } & \multicolumn{3}{|c|}{ Patient reported } \\
\hline & $\begin{array}{l}\text { Low } \\
(n=209)\end{array}$ & $\begin{array}{l}\text { Moderate } \\
(n=219)\end{array}$ & $\begin{array}{l}\text { High } \\
(n=185)\end{array}$ & $\begin{array}{l}\text { Low } \\
(n=209)\end{array}$ & $\begin{array}{l}\text { Moderate } \\
(\mathrm{n}=219)\end{array}$ & $\begin{array}{l}\text { High } \\
(n=185)\end{array}$ \\
\hline $\begin{array}{l}\text { On level ground, I walk slower than people of the same } \\
\text { age because of my breathlessness, or have to stop for } \\
\text { breath when walking at my own pace (grade 2) }\end{array}$ & & & & $64(32.2)$ & $61(29.0)$ & $57(32.6)$ \\
\hline $\begin{array}{l}\text { I stop for breath after walking for a few minutes } \\
\text { On level ground (grade 3) }\end{array}$ & & & & $43(21.6)$ & $31(14.8)$ & $27(15.4)$ \\
\hline $\begin{array}{l}\text { I am too breathless to leave the house or I am } \\
\text { breathless when dressing (grade 4) }\end{array}$ & & & & $9(4.5)$ & 25 ( 11.9$)$ & II (6.3) \\
\hline COPD Assessment Test & & & & $\mathrm{n}=20 \mathrm{I}$ & $\mathrm{n}=210$ & $\mathrm{n}=179$ \\
\hline Mean (SD) & & & & $23.0(7.8)$ & $23.9(7.8)$ & $22.9(7.7)$ \\
\hline COPD Assessment Test (grouped), n (\%) & & & & $n=201$ & $\mathrm{n}=210$ & $\mathrm{n}=179$ \\
\hline $0-9$ & & & & $9(4.5)$ & $13(6.2)$ & $15(8.4)$ \\
\hline $10-20$ & & & & $58(28.9)$ & $67(31.9)$ & $49(27.4)$ \\
\hline $21-30$ & & & & $93(46.3)$ & $85(40.5)$ & $94(52.5)$ \\
\hline$>30$ & & & & $4 \mathrm{I}(20.4)$ & $45(21.4)$ & $21(11.7)$ \\
\hline Jenkins Sleep Evaluation Questionnaire, mean (SD) & & & & $n=194$ & $n=201$ & $\mathrm{n}=174$ \\
\hline & & & & $6.8(5.2)$ & $6.3(5.1)$ & $4.9(4.5)$ \\
\hline
\end{tabular}

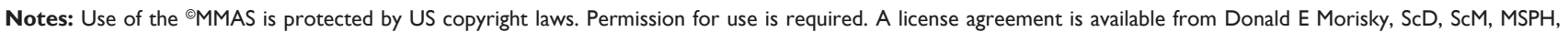
Professor, Department of Community Health Sciences, UCLA School of Public Health, 650 Charles E. Young Drive South, Los Angeles, CA 90095 - I772.

Abbreviation: MMAS-8, Morisky Medication Adherence Scale 8.

Table 4 Physician-reported symptom burden and health-related quality of life (patient record form assessed)

\begin{tabular}{|c|c|c|c|}
\hline & $\begin{array}{l}\text { Primary } \\
\text { study } \\
\text { population } \\
\end{array}$ & $\begin{array}{l}\text { Study } \\
\text { population, no } \\
\text { FEV, criteria } \\
\end{array}$ & $\begin{array}{l}\text { Study population, } \\
\text { dual/triple therapy } \\
\geq 6 \text { months }\end{array}$ \\
\hline Impact of COPD on getting up and ready for the day, $\mathrm{n}(\%)$ & $\mathrm{n}=690$ & $\mathrm{n}=2,95 \mathrm{I}$ & $\mathrm{n}=606$ \\
\hline No impact & $85(12.3)$ & $647(21.9)$ & $73(12.0)$ \\
\hline Rarely impacts & $186(27.0)$ & $945(32.0)$ & I6I (26.6) \\
\hline Occasionally impacts & $224(32.5)$ & $823(27.9)$ & $201(33.2)$ \\
\hline Frequently impacts & $157(22.8)$ & $429(14.5)$ & $136(22.4)$ \\
\hline Constantly impacts & $38(5.5)$ & $107(3.6)$ & $35(5.8)$ \\
\hline Impact of COPD on personal relationships, $n(\%)$ & $\mathrm{n}=683$ & $\mathrm{n}=2,876$ & $\mathrm{n}=600$ \\
\hline No impact & $94(13.8)$ & $631(21.9)$ & $87(14.5)$ \\
\hline Rarely impacts & $225(32.9)$ & I,037 (36. I) & $192(32.0)$ \\
\hline Occasionally impacts & $237(34.7)$ & $834(29.0)$ & $206(34.3)$ \\
\hline Frequently impacts & $107(15.7)$ & $326(11.3)$ & $97(16.2)$ \\
\hline Constantly impacts & $20(2.9)$ & $48(1.7)$ & $18(3.0)$ \\
\hline Impact of COPD on leisure/personal time, $\mathrm{n}(\%)$ & $n=688$ & $\mathrm{n}=2,946$ & $\mathrm{n}=604$ \\
\hline No impact & $38(5.5)$ & $335(11.4)$ & $34(5.6)$ \\
\hline Rarely impacts & $158(23.0)$ & $882(29.9)$ & $135(22.4)$ \\
\hline Occasionally impacts & $246(35.8)$ & $998(33.9)$ & $219(36.3)$ \\
\hline Frequently impacts & $200(29.1)$ & $609(20.7)$ & $174(28.8)$ \\
\hline Constantly impacts & $46(6.7)$ & $122(4.1)$ & $42(7.0)$ \\
\hline Impact of COPD on work (if in work), $n$ (\%) & $n=136$ & $\mathrm{n}=744$ & $\mathrm{n}=1 \mathrm{14}$ \\
\hline No impact & $18(13.2)$ & $153(20.6)$ & $17(14.9)$ \\
\hline Rarely impacts & $38(27.9)$ & $275(37.0)$ & $32(28.1)$ \\
\hline Occasionally impacts & $44(32.4)$ & $203(27.3)$ & $37(32.5)$ \\
\hline Frequently impacts & $28(20.6)$ & $95(12.8)$ & $23(20.2)$ \\
\hline Constantly impacts & $8(5.9)$ & $18(2.4)$ & $5(4.4)$ \\
\hline Impact of COPD on sleep, $\mathrm{n}(\%)$ & $\mathrm{n}=687$ & $n=2,921$ & $\mathrm{n}=603$ \\
\hline No impact & $116(16.9)$ & $640(21.9)$ & $105(17.4)$ \\
\hline Rarely impacts & $229(33.3)$ & I, $103(37.8)$ & $195(32.3)$ \\
\hline Occasionally impacts & $210(30.6)$ & $817(28.0)$ & $189(31.3)$ \\
\hline Frequently impacts & $115(16.7)$ & $318(10.9)$ & $100(16.6)$ \\
\hline Constantly impacts & $17(2.5)$ & $43(1.5)$ & $14(2.3)$ \\
\hline
\end{tabular}

Abbreviation: $\mathrm{FEV}_{1}$, forced expiratory volume in I second. 


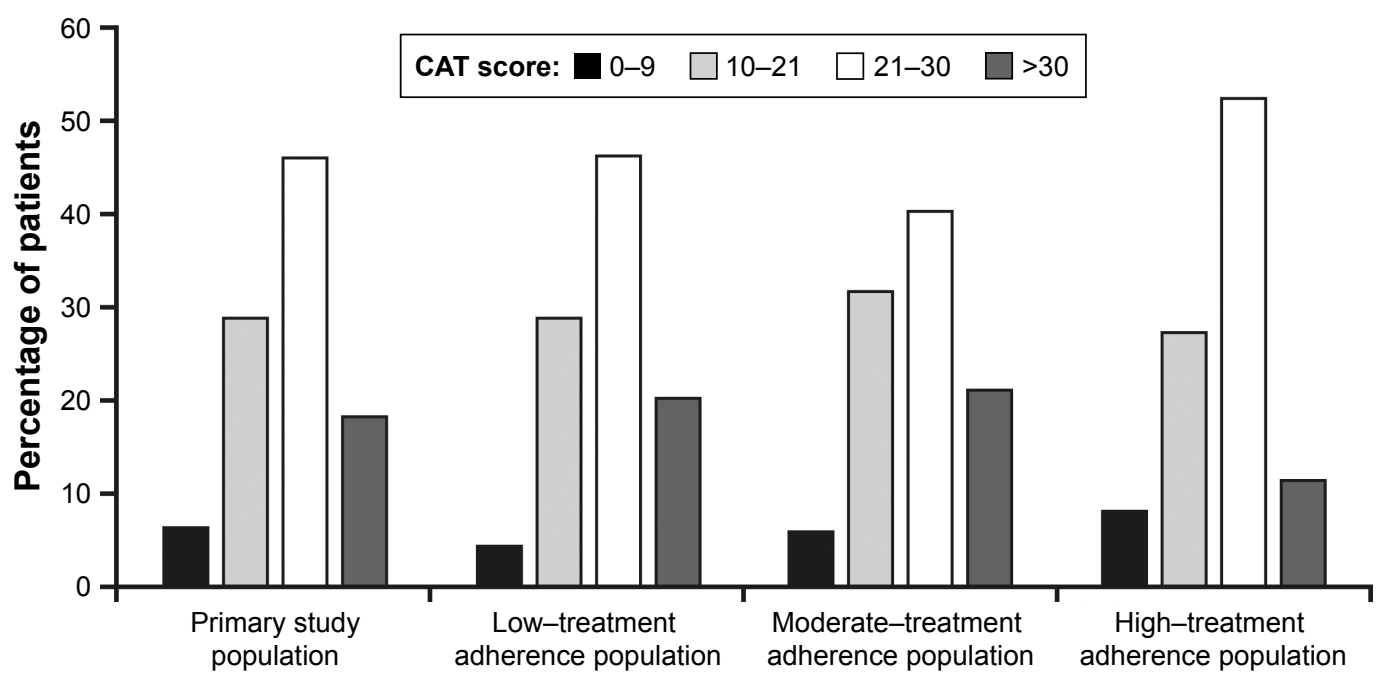

Figure I Percentage of patients by CAT score category for the primary study population overall and by adherence to treatment categories. ${ }^{a}$

Notes: assessed using the MMAS-8: low, <6; moderate, 6-7; high, 8. Use of the ${ }^{\circ M M A S}$ is protected by US copyright laws. Permission for use is required. A license agreement is available from Donald E Morisky, ScD, ScM, MSPH, Professor, Department of Community Health Sciences, UCLA School of Public Health, 650 Charles E. Young Drive South, Los Angeles, CA 90095-1772.

Abbreviations: CAT, COPD Assessment Test; MMAS-8, Morisky Medication Adherence Scale 8.

The use of dual and triple therapy treatment has been associated with clinically meaningful improvements in lung function and HRQOL and reduced exacerbations in patients with COPD. ${ }^{2,3}$ However, we found that despite receiving dual or triple therapy, some patients with $\mathrm{COPD}$ and $\mathrm{FEV}_{1} \leq 65 \%$ reported a significant burden of symptoms that affected their everyday lives. This symptom burden was also significant in those with good treatment adherence. More than half of this patient population presented with CAT scores $>20(64.4 \%)$ and mMRC dyspnea scale scores $\geq 2$ (56.3\%). This large symptom burden was experienced by patients regardless of their lung function and by those who had been receiving treatment for a minimum of 6 months.

Previous studies examining the burden of symptoms for patients with moderate/severe COPD have reported similar results to our findings, such as approximately half of the patients having an mMRC dyspnea scale score $\geq 2$ and associated greater CAT score. ${ }^{16,17}$ However, these studies

Table 5 Symptom burden of patients categorized by GOLD ABCD group

\begin{tabular}{|c|c|c|c|c|c|}
\hline & \multirow{2}{*}{$\begin{array}{l}\text { Overall }^{a} \\
(N=640)\end{array}$} & \multicolumn{4}{|c|}{ GOLD group ${ }^{2}$} \\
\hline & & $\begin{array}{l}A \\
(n=28)\end{array}$ & $\begin{array}{l}B \\
(n=268)\end{array}$ & $\begin{array}{l}C \\
(n=15)\end{array}$ & $\begin{array}{l}D \\
(n=329)\end{array}$ \\
\hline CAT score & $\mathrm{N}=640$ & $\mathrm{n}=28$ & $n=268$ & $n=15$ & $n=329$ \\
\hline Mean (SD) & $23.1(7.9)$ & $6.3(2.5)$ & $21.9(6.4)$ & $7.1(2.0)$ & $26.2(6.4)$ \\
\hline $0-9$, n (\%) & $43(6.7)$ & $28(100.0)$ & $0(0)$ & $15(100.0)$ & $0(0)$ \\
\hline I0-20, n (\%) & $184(28.7)$ & $0(0)$ & $113(42.2)$ & $0(0)$ & 7I (2I.6) \\
\hline $21-30, \mathrm{n}(\%)$ & $294(45.9)$ & $0(0)$ & $128(47.8)$ & $0(0)$ & $166(50.5)$ \\
\hline$>30, \mathrm{n}(\%)$ & $119(18.6)$ & $0(0)$ & $27(10.1)$ & $0(0)$ & $92(28.0)$ \\
\hline mMRC dyspnea scale, $n$ (\%) & $\mathrm{n}=610$ & $\mathrm{n}=27$ & $\mathrm{n}=255$ & $n=12$ & $n=316$ \\
\hline I only get breathless with strenuous exercise (grade 0 ) & $59(9.7)$ & $16(59.3)$ & $26(10.2)$ & $3(25.0)$ & $14(4.4)$ \\
\hline I get short of breath when hurrying on level ground or walking up a slight hill (grade I) & $206(33.8)$ & $8(29.6)$ & $108(42.4)$ & $6(50.0)$ & $84(26.6)$ \\
\hline On level ground, I walk slower than people of the same age because of my & $194(31.8)$ & $2(7.4)$ & $80(31.4)$ & $3(25.0)$ & $109(34.5)$ \\
\hline \multicolumn{6}{|l|}{ breathlessness, or have to stop for breath when walking at my own pace (grade 2) } \\
\hline I stop for breath after walking for a few minutes on level ground (grade 3) & $107(17.5)$ & I (3.7) & $35(13.7)$ & $0(0)$ & $71(22.5)$ \\
\hline I am too breathless to leave the house or I am breathless when dressing (grade 4) & $44(7.2)$ & $0(0)$ & $6(2.4)$ & $0(0)$ & $38(12.0)$ \\
\hline JSEQ score, $\mathrm{n}$ & $\mathrm{n}=589$ & $\mathrm{n}=27$ & $\mathrm{n}=244$ & $n=13$ & $\mathrm{n}=305$ \\
\hline Mean (SD) & $6.0(5.0)$ & I.I (2.0) & $4.9(4.4)$ & I.I (I.4) & $7.6(5.1)$ \\
\hline
\end{tabular}

Note: ${ }^{a}$ Overall GOLD group.

Abbreviations: CAT, COPD Assessment Test; GOLD, Global Initiative for Chronic Obstructive Lung Disease; JSEQ, Jenkins Sleep Evaluation Questionnaire; mMRC, Modified Medical Research Council. 
have often been limited to national data or evaluation of a limited number of symptoms. ${ }^{16-19}$ Uniquely, this study used treatment as a criterion for analyses of symptom burden applied to a worldwide patient population. The findings highlight the large unmet need for further treatments for symptom control in patients with COPD, whose disease significantly affects their daily lives despite receiving current standard-ofcare therapy for severe disease. The disease characteristics and symptom burden for the secondary study populations (those without an $\mathrm{FEV}_{1}$ inclusion criterion and those who had been on medication for a minimum of 6 months) were similar to those of the primary study population. These similarities demonstrate that the large burden of symptoms for patients with COPD is not restricted to those with reduced lung function, those with suboptimal adherence to treatment, or those who have been on dual or triple therapy for $<6$ months.

In this study population, symptoms occurred most frequently during daytime hours and the autumn and winter months. These results are consistent with previous studies. The ASSESS study reported that despite regular treatment, $90.5 \%$ of patients with stable COPD experienced symptoms at some point during the day, ${ }^{20}$ while the TORCH long-term international study demonstrated an increased risk of COPD exacerbations in the winter months in northern (Canada, China, 26 eastern and western European countries, and the USA) and southern (Argentina, Australia, Brazil, Chile, New Zealand, and South Africa) regions. ${ }^{21}$ Our results on the prevalence of daytime/nighttime symptoms, breathlessness being the most common symptom, prevalence of sleep impairment, and more than half of patients being dissatisfied with their current medications' ability to control symptoms were in general agreement with previous studies. ${ }^{4,17}$

Previous studies have reported that concordance of symptom burden and impact on HRQOL reports from physicians and patients are greater for patients with more severe COPD compared with those with milder disease. ${ }^{21}$ In our study, there was reasonable concordance between physician- and patient-reported responses describing symptom burden, suggesting that physician-patient communication is not a barrier to improving care for patients with more severe disease, although it may be a factor for those with milder disease.

The data used in this study were collected from patients consulting their physicians in a routine care setting with no excessively restrictive exclusion criteria and, therefore, are representative of patients in a real-world clinical setting. However, this study has some potential limitations. Patients who consulted a physician and agreed to participate were included in the study; therefore, patients who consulted their physician more frequently or who were experiencing COPD-related symptoms may be overrepresented in the study population. Furthermore, the impact of undertreatment on symptom burden could not be evaluated and the focus of this study was symptom burden, and exacerbation rate was not included; therefore, only one dimension of the clinical burden was considered for patients with COPD. For these reasons, generalizability of our findings to the whole COPD population may be limited. It is also not known to what extent those patients who did not complete a patient self-completion questionnaire (and were therefore excluded from the analysis) differed from those who did complete the questionnaire; this circumstance may introduce a further bias of patient selection. In addition, the quality of data is dependent on accurate reporting of information by physicians and patients, although physicians were permitted to use patient records to limit errors in actual treatment, events, and comorbidities. As part of sensitivity analyses, the GOLD ABCD classification system was used to categorize patients based on their degrees of symptom burden and exacerbation risk. However, the timing of classification, whether at diagnosis or subsequent evaluations, may be subject to interpatient variation. Other limitations include the cross-sectional retrospective study design. Our report assessed associations between factors and did not identify causality, and retrospective reports of adverse events were not provided. We also did not assess the effect of patients receiving double or triple therapy on their symptom burden, which may further limit the interpretation of our results.

\section{Conclusion}

Despite receiving dual or triple therapy, a number of patients with COPD still reported major symptoms that affected their everyday lives and impaired their health status. For patients with COPD receiving dual or triple therapy, we found associations between symptomatic burden and adherence to treatment. Nevertheless, some highly adherent patients still had substantial symptom burden. Further research is necessary to identify novel treatment strategies for patients who continue to experience symptoms despite receiving dual or triple treatment regimens.

\section{Acknowledgments}

This analysis was funded by AstraZeneca. We wish to thank Tom Bailey and Megan Scott for their contributions to this work. Editorial support was provided by Debra Scates, $\mathrm{PhD}$, of JK Associates, Inc. and Michael A Nissen, ELS, of AstraZeneca. This support was funded by AstraZeneca. 


\section{Disclosure}

Mark Small is an employee of Adelphi Real World. Stephanie Chen, Leandro Lindner, and Xiao Xu are employees of AstraZeneca. The authors report no other conflicts of interest in this work.

\section{References}

1. Lopez-Campos JL, Tan W, Soriano JB. Global burden of COPD. Respirology. 2016;21(1):14-23.

2. GOLD [webpage on the Internet]. GOLD 2018 Global Strategy for the Diagnosis, Management and Prevention of COPD. Available from: http:// goldcopd.org/gold-reports/. Accessed March 20, 2018.

3. Tabberer M, Lomas DA, Birk R, et al. Once-daily triple therapy in patients with COPD: patient-reported symptoms and quality of life. Adv Ther. 2018;35(1):56-71.

4. Singh D, Miravitlles M, Vogelmeier C. Chronic obstructive pulmonary disease individualized therapy: tailored approach to symptom management. Adv Ther. 2017;34(2):281-299.

5. Anderson P, Benford M, Harris N, Karavali M, Piercy J. Real-world physician and patient behaviour across countries: Disease-Specific Programmes-a means to understand. Curr Med Res Opin. 2008;24(11): 3063-3072.

6. ICC/ESOMAR. International Code on Market and Social Research 2007. Available from: https://www.esomar.org/uploads/public/knowledgeand-standards/codes-and-guidelines/ICCESOMAR_Code_English_. pdf. Accessed November 7, 2017.

7. Morisky DE, Ang A, Krousel-Wood M, Ward HJ. Predictive validity of a medication adherence measure in an outpatient setting. J Clin Hypertens (Greenwich). 2008;10(5):348-354.

8. Morisky DE, DiMatteo MR. Improving the measure of self-reported medication nonadherence: final response. J Clin Epidemiol. 2011; 64(3):258-263.

9. Krousel-Wood MA, Islam T, Webber LS, Re RS, Morisky DE, Muntner P. New medication adherence scale versus pharmacy fill rates in seniors with hypertension. Am J Manag Care. 2009;15(1):59-66.
10. Jones PW, Harding G, Berry P, et al. Development and first validation of the COPD Assessment Test. Eur Respir J. 2009;34(3):648-654.

11. Williams N. The MRC breathlessness scale. Occup Med (Lond). 2017; 67(6):496-497.

12. Jenkins CD, Stanton BA, Niemcryk SJ, Rose RM. A scale for the estimation of sleep problems in clinical research. $J$ Clin Epidemiol. 1988;41(4):313-321.

13. Stata Statistical Software: Release 14. College Station, TX: StataCorp LP. [Computer program] 2015.

14. Ghobadi H, Ahari SS, Kameli A, Lari SM. The relationship between COPD Assessment Test (CAT) scores and severity of airflow obstruction in stable COPD patients. Tanaffos. 2012;11(2):22-26.

15. Stenton C. The MRC breathless scale. Occup Med (Lond). 2008;58(3): 226-227.

16. Henoch I, Strang S, Lofdahl CG, Ekberg-Jansson A. Health-related quality of life in a nationwide cohort of patients with COPD related to other characteristics. Eur Clin Respir J. 2016;3:31459.

17. Miravitlles M, Ribera A. Understanding the impact of symptoms on the burden of COPD. Respir Res. 2017;18(1):67.

18. Joshi M, Joshi A, Bartter T. Symptom burden in chronic obstructive pulmonary disease and cancer. Curr Opin Pulm Med. 2012;18(2):97-103.

19. Ding B, DiBonaventura M, Karlsson N, Bergstrom G, Holmgren U. A cross-sectional assessment of the burden of COPD symptoms in the US and Europe using the National Health and Wellness Survey. Int J Chron Obstruct Pulmon Dis. 2017;12:529-539.

20. Miravitlles M, Worth H, Soler Cataluna JJ, et al. Observational study to characterise 24-hour COPD symptoms and their relationship with patient-reported outcomes: results from the ASSESS study. Respir Res. 2014;15:122.

21. Jenkins CR, Celli B, Anderson JA, et al. Seasonality and determinants of moderate and severe COPD exacerbations in the TORCH study. Eur Respir J. 2012;39(1):38-45.
International Journal of COPD

\section{Publish your work in this journal}

The International Journal of COPD is an international, peer-reviewed journal of therapeutics and pharmacology focusing on concise rapid reporting of clinical studies and reviews in COPD. Special focus is given to the pathophysiological processes underlying the disease, intervention programs, patient focused education, and self management protocols.

\section{Dovepress}

This journal is indexed on PubMed Central, MedLine and CAS. The manuscript management system is completely online and includes a very quick and fair peer-review system, which is all easy to use. Visit http://www.dovepress.com/testimonials.php to read real quotes from published authors. 